\title{
Changes in Renal Function in Elderly Patients Following Intravenous Iodinated Contrast Administration: A Retrospective Study
}

\author{
Ali Alsafi, Zaid Alsafi, Amish Lakhani, and Nicola H. Strickland \\ Radiology Department, Imperial College Healthcare NHS, Hammersmith Hospital, Du Cane Road, London W12 0HS, UK \\ Correspondence should be addressed to Ali Alsafi; ali.alsafi03@alumni.imperial.ac.uk
}

Received 12 January 2014; Revised 19 February 2014; Accepted 25 February 2014; Published 24 March 2014

Academic Editor: Keiji Tanimoto

Copyright (c) 2014 Ali Alsafi et al. This is an open access article distributed under the Creative Commons Attribution License, which permits unrestricted use, distribution, and reproduction in any medium, provided the original work is properly cited.

Background. Contrast-induced nephropathy (CIN) is a recognised complication of intravascular administration of iodinated contrast media (ICM). Previous studies suggest a higher incidence in the elderly, but no large study has assessed this to date. We set out to assess changes in creatinine in elderly inpatients following computed tomography (CT) examination and compare those who received intravenous contrast to those who did not. Methods. Using the Radiology Information System in two teaching hospitals, inpatients over the age of seventy who had a CT examination and a baseline creatinine were identified and their follow-up creatinine levels were analysed. Elderly inpatients who underwent a noncontrast CT over the same period were used as controls. Results. 677 elderly inpatients who received ICM were compared with 487 controls. 9.2\% of patients who received ICM developed acute kidney injury (AKI) compared to $3.5 \%$ of inpatient controls $(P<0.0001)$. Patients with higher baseline eGFR had a higher incidence of post-CT AKI. Conclusions. The incidence of post-CT AKI is higher in patients who received IV ICM compared to those who did not; the difference may be partly attributable to contrast-induced nephropathy. This suggests that the incidence of $\mathrm{CIN}$ in the elderly may not be as high as previously thought.

\section{Background}

Intravascular administration of iodinated contrast media (ICM) is used in a variety of diagnostic and therapeutic procedures. While ICM are generally safe, only resulting in minor side effects, they can result acute renal impairment known as contrast induced nephropathy (CIN) [1]. The majority of cases of CIN are self-limiting, although occasionally chronic renal failure ensues necessitating long-term renal replacement. Even when transient, CIN is associated with increased length of hospital stay and significant morbidity $[1,2]$.

Diabetes mellitus, preexisting renal failure, congestive heart failure, and hypotension, dehydration as well as the use of ACE inhibitors, diuretics, and nonsteroidal antiinflammatory drugs have all been identified as important risk factors for developing $\mathrm{CIN}[3,4]$. Hydration in high-risk patients remains the most important preventative measure $[5,6]$.

Many attempts at defining CIN exist, but the most widely accepted definition is a $25 \%$ rise in serum creatinine concentration from baseline or an absolute increase by $44 \mu \mathrm{mol} / \mathrm{L}$ over the 48 to 72 hours following ICM administration, once other causes of renal impairment have been excluded $[2,4]$.

The use of CT in clinical medicine has exponentially increased over the past two decades, including managing elderly patients, who are thought to be particularly susceptible to developing CIN [7-10]. The true incidence of CIN in the elderly is not known, as most studies addressing the issue assumed that acute kidney injury (AKI) following ICM administration is in fact CIN [11-13]. The results of these studies are therefore likely to be an overestimate, as they failed to compare the incidence of AKI in their patient population to that of a control group. AKI itself is difficult to define, 
but a practical definition is that of a rapid deterioration in renal function resulting in the inability to maintain fluid, electrolyte, or acid-base homeostasis [14].

CIN has come recently under question as a clinical entity, with suggestions that its incidence and significance have been grossly overestimated. One study even questions the existence of CIN, particularly in patients with preexisting normal renal function [15-17].

In this study, we sought to retrospectively evaluate changes in renal function in elderly patients who underwent a contrast enhanced CT examination and compare to controls who had a noncontrast CT. The aim was to estimate the incidence of post-CT AKI in the elderly.

\section{Methods}

Using the Radiology Information System (RIS) in two teaching hospitals, inpatients over the age of seventy who had a contrast enhanced computed tomography (CT) examination between August 2011 and August 2012 were identified. Demographic and examination data were recorded. Using the hospitals' pathology results systems, the most recent creatinine $(\mathrm{Cr})$ values prior to the contrast examination were recorded. Follow-up Cr values 48-72 hours following the examination were also noted. The most recent $\mathrm{Cr}$ prior to the CT examination (up to six weeks) was taken as the baseline against which changes in $\mathrm{Cr}$ were compared. eGFR was calculated using the modified diet in renal disease (MDRD) formula [18].

Information relating to prehydration, posthydration, and administration of NAC was not readily available and not recorded, although our standard in-hospital practice is to preand posthydrate high-risk patients.

We excluded patients who had no baseline creatinine, those who had no blood tests between 48 and 72 hours following the CT examination, and those on haemodialysis, peritoneal dialysis, or hemofiltration. Patients who had a nephrostomy or renal stent placement within 7 days of the study were also excluded. Patients who had a contrast head CT receiving $50 \mathrm{~mL}$ of Omnipaque 300 were excluded. This group constitutes a small number of patients with a significantly lower contrast dose to the remainder of the study population.

All elderly inpatients who underwent a noncontrast examination over the same period were used as controls with the same exclusion criteria. These patients were also identified using the RIS system. Those who developed AKI of an order comparable to that seen in CIN, (25\% rise in serum creatinine concentration from baseline or an absolute increase by $44 \mu \mathrm{mol} / \mathrm{L}$ ) were identified. The incidence of AKI was calculated for the ICM and control group.

Institutional review board approval was obtained and informed consent was waived prior to commencing this retrospective study.

2.1. Statistical Analysis. Two-tailed Fisher's exact test was used to compare categorical data, while two-tailed Student's $t$-test was used to compare numerical data. $P$ values $<0.05$ were considered significant. Odds ratio was calculated using
TABLE 1: Demographic data and baseline eGFR $\left(\mathrm{mL} / \mathrm{min} / 1.73 \mathrm{~m}^{2}\right)$.

\begin{tabular}{lccc}
\hline & $\begin{array}{c}\text { Body CT } \\
(n=677)\end{array}$ & $\begin{array}{c}\text { Controls } \\
(n=487)\end{array}$ & $P$ value \\
\hline Average age (range) & $80.4(70-102)$ & $82(70-103)$ & $<0.0001^{*}$ \\
Males (\%) & $340(50.2 \%)$ & $240(49.3 \%)$ & $0.7666^{\#}$ \\
Females (\%) & $337(49.8 \%)$ & $247(50.7 \%)$ & \\
Baseline eGFR > 90 & $220(32 \%)$ & $153(31.4 \%)$ & $0.7030^{\#}$ \\
\hline
\end{tabular}

${ }^{*}$ Unpaired Student's $t$-test; ${ }^{\#}$ Fisher's exact test.

TABle 2: Number of patients developing post-CT AKI in the ICM and control groups with varying baseline eGFR. (eGFR in $\left.\mathrm{mL} / \mathrm{min} / 1.73 \mathrm{~m}^{2}\right)$.

\begin{tabular}{lcc}
\hline Baseline eGFR & ICM group (\%) & Controls (\%) \\
\hline$>90$ & $13(5.91 \%)(n=220)$ & $2(1.31 \%)(n=153)$ \\
$60-90$ & $19(9.13 \%)(n=208)$ & $2(1.23 \%)(n=162)$ \\
$45-60$ & $7(7.37 \%)(n=95)$ & $3(4.48 \%)(n=67)$ \\
$<45$ & $23(14.9 \%)(n=154)$ & $10(9.52 \%)(n=105)$ \\
\hline Total & $62(9.16 \%)(n=677)$ & $17(3.49 \%)(n=487)$ \\
\hline
\end{tabular}

GraphPad Prism 5.0 for Mac OS X (GraphPad Software Inc., San Diego, CA, USA).

\section{Results and Discussion}

920 inpatients over the age of 70 had a contrast enhanced CT examination between August 2011 and 2012, including 825 body CT examinations and 95 head CT examinations; the latter were excluded. A further 81 patients were excluded because they were receiving renal replacement therapy and one patient was excluded because of a recent nephrostomy.

677 patients had a baseline creatinine and at least one further creatinine measurement 48-72 hours following their CT. None of the patients had more than one contrast enhanced examination during the study period. Over the same period, 850 inpatients underwent a CT examination without contrast. 363 of these patients were excluded: 17 because they were receiving renal replacement, 48 who had no baseline creatinine, one who had acute kidney injury predating the CT examination, and 297 who did not have a creatinine level measured 48-72 hours following their CT examination. The remaining 487 cases were used as controls. There was no significant difference in sex or baseline eGFR between the two groups, although the control group was slightly older than the ICM group. (Demographics and baseline eGFR are shown in Tables 1 and 2 resp.)

In the 48 to 72 hours following intravenous administration of $80-120 \mathrm{~mL}$ of Omnipaque $300,5.9 \%(13 / 220)$ of patients with a baseline eGFR $>90 \mathrm{~mL} / \mathrm{min} / 1.73 \mathrm{~m}^{2}$ developed AKI compared with 9.1\% (19/208), 7.4\% (7/95), and $14.9 \%(23 / 154)$ of those with eGFR $60-90 \mathrm{~mL} / \mathrm{min} / 1.73 \mathrm{~m}^{2}$, $45-60 \mathrm{~mL} / \mathrm{min} / 1.73 \mathrm{~m}^{2}$, and $<45 \mathrm{~mL} / \mathrm{min} / 1.73 \mathrm{~m}^{2}$, respectively (Figure 1). The relative risk for developing post-CT AKI in patients who had $80-120 \mathrm{~mL}$ of ICM with a baseline eGFR $<45 \mathrm{~mL} / \mathrm{min} / 1.73 \mathrm{~m}^{2}$ compared to those with an 


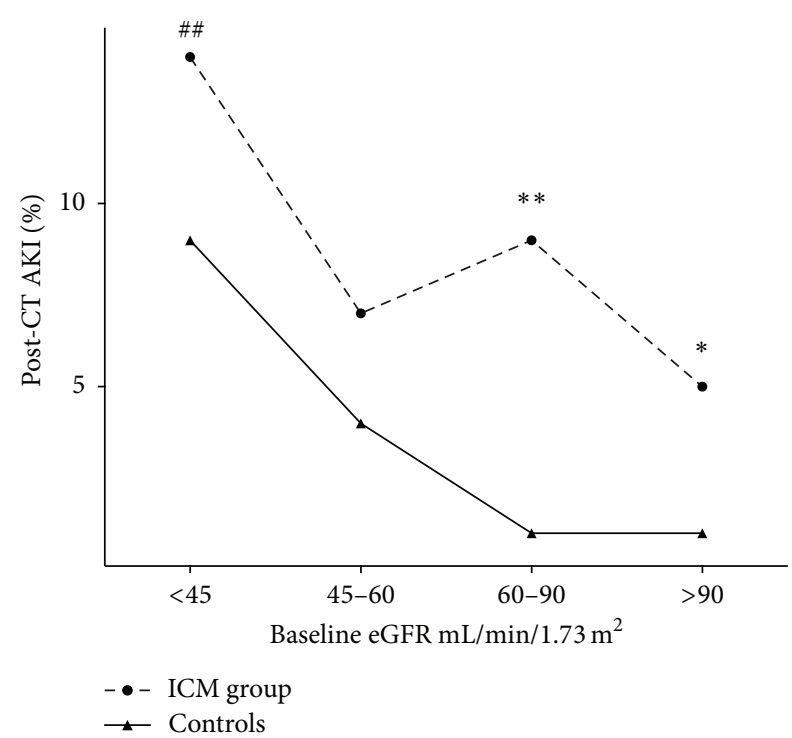

FIGURE 1: Percentage of patients developing post-CT AKI in the ICM group compared to controls. Using Fisher's exact test: ${ }^{*} P<$ 0.05 and ${ }^{* *} P<0.001$, comparing the ICM group versus controls and ${ }^{\# \#} P<0.005$ comparing to patients with a baseline GFR $>$ $90 \mathrm{~mL} / \mathrm{min} / 1.73 \mathrm{~m}^{2}$.

eGFR > $90 \mathrm{~mL} / \mathrm{min} / 1.73 \mathrm{~m}^{2}$ was 1.65 (95\% CI 1.25 to 2.18 , $P=0.0043)$.

The relative risk for developing post-CT AKI in inpatients who had $80-120 \mathrm{~mL}$ of ICM compared with controls is 1.35 (95\% CI 1.22 to $1.57, P<0.0001$ ).

Intravenous contrast media (ICM) are widely used in diagnostic and interventional radiology; their use, however, is associated with up to $11 \%$ of hospital-acquired AKI [5]. CIN is a cause of significant morbidity and mortality in hospital patients and is thought to be an important cause of AKI in this population alongside sepsis, hypoperfusion, major surgery, and nephrotoxic medication $[5,11-13]$.

Increasing age is an independent predictor for developing CIN with a fivefold increase in risk in patients over the age of seventy [7].

Previous studies, including MacDonald et al's recent large meta-analysis, showed no difference in incidence of postCT AKI, between contrast and control groups. These studies assessed significantly younger patient populations than ours (median patient age of 62 versus 82 in our study) and their results may not be generalizable to an older age group $[15,16$, 19].

Davenport et al. on the other hand demonstrated that IV ICM is an important risk factor, albeit not the only one, in developing post-CT AKI in an adult patient population [17]. Our results extend these conclusions to a significantly older population, which may be even more susceptible to CIN.

Two studies estimated the incidence of CIN in the elderly at $6 \%$ and $14 \%$, respectively. Neither study accounted for other causes of AKI nor did they compare with controls $[9,10]$. To our knowledge, this is the first study to compare the incidence of post-CT AKI in elderly patients who received IV ICM with controls who had nonenhanced examinations.

While the excess AKI in the ICM group is, at least partly, likely attributable to contrast administration, there may be inherent differences between the two groups, with patients not receiving ICM being perceived as high-risk and therefore undergoing noncontrast examinations. The fact that the average baseline eGFR, age, and gender distribution are similar in the two groups does not support this.

Furthermore, a significant number of the noncontrast studies were head CT examinations (87\%), suggesting that the disease processes for which the two groups presented to hospital may be different, with the ICM group being more likely to have systemic, rather than neurological, symptoms and therefore more likely to develop AKI. This is merely speculative but may partly account for the differences seen in conjunction with true CIN. If this is the case, the incidence of CIN may even be lower.

\section{Limitations}

Although we did not assess for risk factors other than preexisting renal impairment, by using a control population with similar demographics who were inpatients during the same study period, the difference in incidence of AKI may be partly attributable to the administration of IV ICM.

Our results apply to an elderly hospital inpatient population and may not be generalizable to a healthier outpatient population, whose risk of CIN is perhaps even lower.

\section{Conclusions}

The incidence of AKI in our elderly inpatient population was $9.2 \%$ after administration of $80-120 \mathrm{~mL}$ of intravenous ICM compared with 3.5\% in inpatient controls. The difference between the two groups may be, at least partly, attributable to ICM. Caution, however, needs to be exercised in elderly patients with low baseline eGFR $\left(<45 \mathrm{~mL} / \mathrm{min} / 1.73 \mathrm{~m}^{2}\right)$ as post-CT AKI approaches $15 \%$ in this group.
Abbreviations
CIN: Contrast induced nephropathy
ICM: Iodinated contrast media
CT: $\quad$ Computed tomography
AKI: Acute kidney injury
eGFR: estimated glomerular filtration rate
Cr: Creatinine
NAC: n-Acetylcysteine
MDRD: Modified diet in renal disease
RIS: The Radiology Information System.

\section{Conflict of Interests}

The authors declare that there is no conflict of interests regarding the publication of this paper. 


\section{References}

[1] P. Parfrey, "The clinical epidemiology of contrast-induced nephropathy," CardioVascular and Interventional Radiology, vol. 28, supplement 2, pp. S3-S11, 2005.

[2] J. Richenberg, "How to reduce nephropathy following contrastenhanced CT: a lesson in policy implementation," Clinical Radiology, vol. 67, no. 12, pp. 1136-1145, 2012.

[3] P. A. McCullough and K. R. Sandberg, "Epidemiology of contrast-induced nephropathy," Reviews in Cardiovascular Medicine, vol. 4, supplement 5, pp. S3-S9, 2003.

[4] R. Mehran and E. Nikolsky, "Contrast-induced nephropathy: definition, epidemiology, and patients at risk," Kidney International, vol. 69, supplement 100, pp. S11-S15, 2006.

[5] C. E. A. Balemans, L. J. M. Reichert, B. I. H. van Schelven, J. A. J. G. van den Brand, and J. F. M. Wetzels, "Epidemiology of contrast material-induced nephropathy in the era of hydration," Radiology, vol. 263, no. 3, pp. 706-713, 2012.

[6] Acute Kidney Injury, http://www.renal.org/clinical/guidelinessection/AcuteKidneyInjury.aspx.

[7] S. L. Chen, J. Zhang, F. Yei et al., "Clinical outcomes of contrastinduced nephropathy in patients undergoing percutaneous coronary intervention: a prospective, multicenter, randomized study to analyze the effect of hydration and acetylcysteine," International Journal of Cardiology, vol. 126, no. 3, pp. 407-413, 2008.

[8] R. Mehran, E. D. Aymong, E. Nikolsky et al., "A simple risk score for prediction of contrast-induced nephropathy after percutaneous coronary intervention: development and initial validation," Journal of the American College of Cardiology, vol. 44, no. 7, pp. 1393-1399, 2004.

[9] A. M. Mitchell, A. E. Jones, J. A. Tumlin, and J. A. Kline, "Prospective study of the incidence of contrast-induced nephropathy among patients evaluated for pulmonary embolism by contrast-enhanced computed tomography," Academic Emergency Medicine, vol. 19, no. 6, pp. 618-625, 2012.

[10] M. J. Laugharne, M. Paravasthu, A. Preston, and K. O. Hill, "CT pulmonary angiography in elderly patients: Outcomes in patients aged $>85$ years," Clinical Radiology, vol. 68, no. 5, pp. 449-454, 2013.

[11] F. G. Brivet, D. J. Kleinknecht, P. Loirat, and P. J. M. Landais, "Acute renal failure in intensive care units-causes, outcome, and prognostic factors of hospital mortality: a prospective, multicenter study," Critical Care Medicine, vol. 24, no. 2, pp. 192$198,1996$.

[12] S. S. Waikar, K. D. Liu, and G. M. Chertow, "Diagnosis, epidemiology and outcomes of acute kidney injury," Clinical Journal of the American Society of Nephrology, vol. 3, no. 3, pp. 844-861, 2008.

[13] K. Nash, A. Hafeez, and S. Hou, "Hospital-acquired renal insufficiency," The American Journal of Kidney Diseases, vol. 39, no. 5, pp. 930-936, 2002.

[14] Acute Kidney Injury, http://www.renal.org/guidelines/modules.

[15] R. J. McDonald, J. S. McDonald, J. P. Bida et al., "Intravenous contrast material-induced nephropathy: causal or coincident phenomenon?" Radiology, vol. 267, no. 1, pp. 106-118, 2013.

[16] J. S. McDonald, R. J. McDonald, J. Comin et al., "Frequency of acute kidney injury following intravenous contrast medium administration: a systematic review and meta-analysis," Radiology, vol. 267, no. 1, pp. 119-128, 2013.
[17] M. S. Davenport, S. Khalatbari, J. R. Dillman, R. H. Cohan, E. M. Caoili, and J. H. Ellis, "Contrast material-induced nephrotoxicity and intravenous low-osmolality iodinated contrast material," Radiology, vol. 267, no. 1, pp. 94-105, 2013.

[18] A. S. Levey, T. Greene, J. W. Kusek, G. J. Beck, and M. S. Group, "A simplified equation to predict glomerular filtration rate from serum creatinine," Journal of the American Society of Nephrology, vol. 11, article A0828, 2000.

[19] R. J. Bruce, A. Djamali, K. Shinki, S. J. Michel, J. P. Fine, and M. A. Pozniak, "Background fluctuation of kidney function versus contrast-induced nephrotoxicity," The American Journal of Roentgenology, vol. 192, no. 3, pp. 711-718, 2009. 


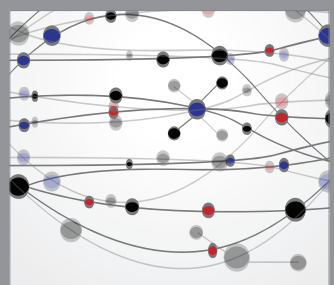

The Scientific World Journal
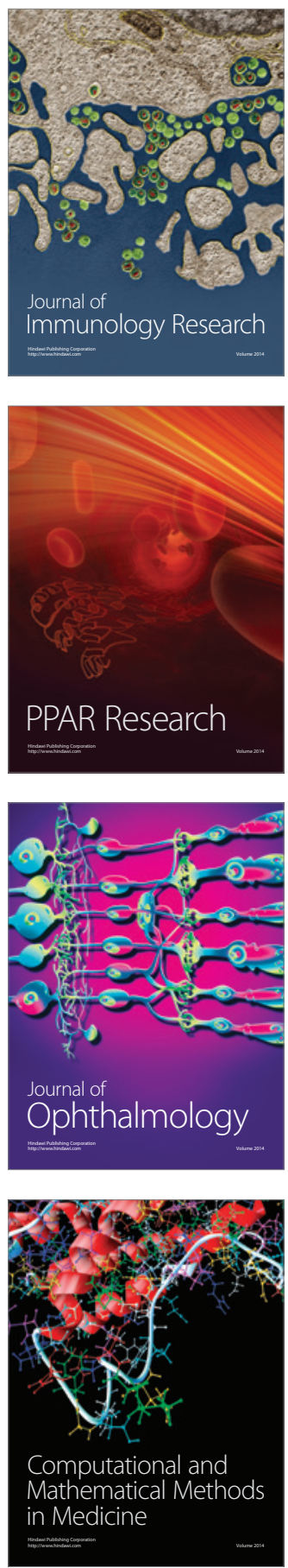

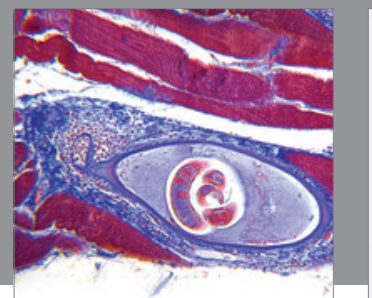

Gastroenterology

Research and Practice
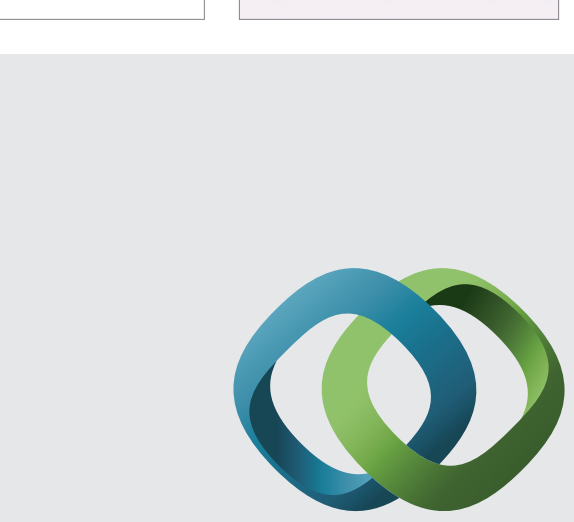

\section{Hindawi}

Submit your manuscripts at

http://www.hindawi.com
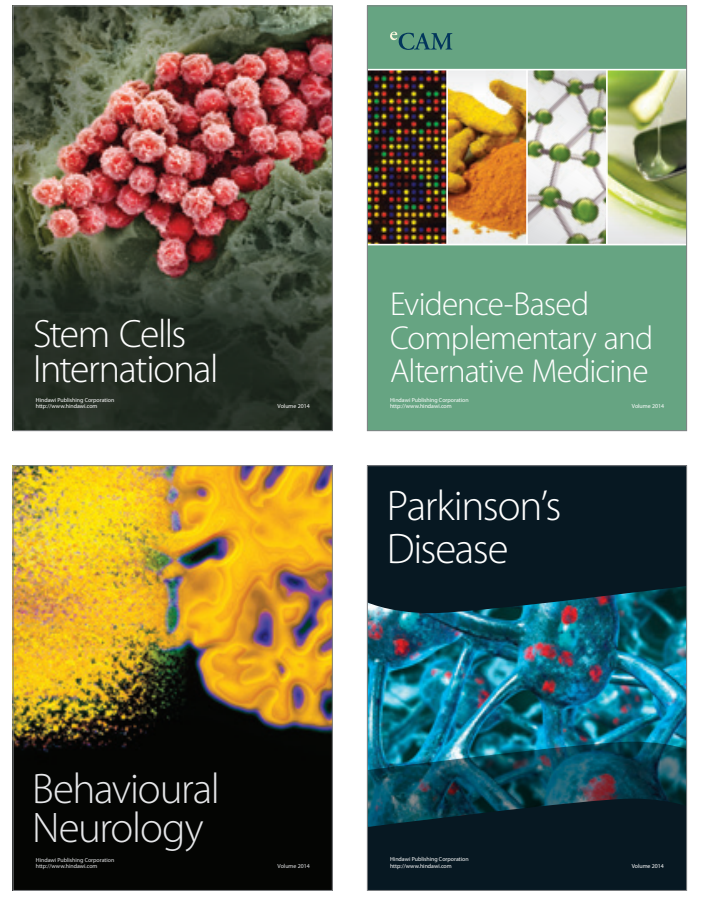
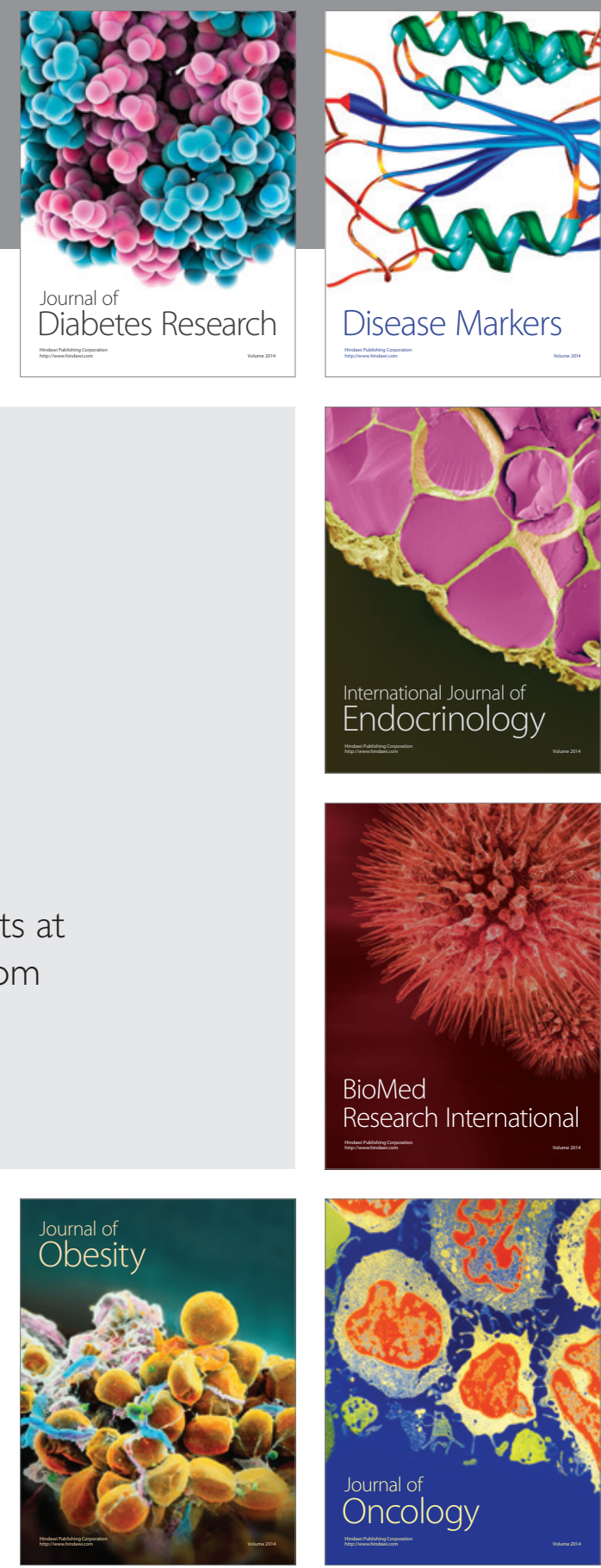

Disease Markers
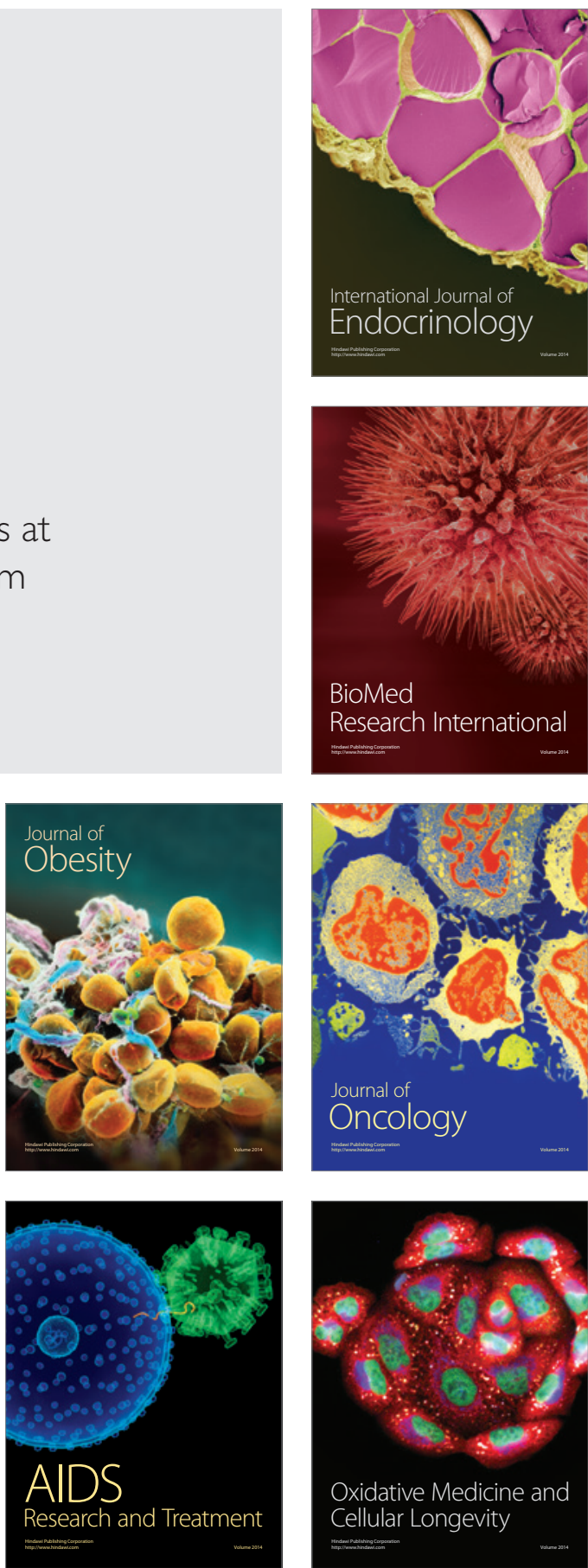